\title{
On the signature of tensile blobs in the scattering function of a stretched polymer
}

\author{
Carlo Pierleoni \\ INFM and Dipartimento di Fisica, Università degli Studi, L'Aquila (Italy) \\ Gianluigi Arialdi and Jean-Paul Ryckaert \\ Département de Physique, Université Libre de Bruxelles, Brussels (Belgium)
}

(October 7, 2018)

\begin{abstract}
We present Monte Carlo data for a linear chain with excluded volume subjected to a uniform stretching. Simulation of long chains (up to 6000 beads) at high stretching allows us to observe the signature of tensile blobs as a crossover in the scaling behavior of the chain scattering function for wave vectors perpendicular to stretching. These results and corresponding ones in the stretching direction allow us to verify for the first time Pincus prediction on scaling inside blobs. Outside blobs, the scattering function is well described by the Debye function for a stretched ideal chain.
\end{abstract}

61.25.Hq,83.20.Jp

The notion of blobs in polymer physics is a widespread concept which indicates a change of fractal dimensionality of the polymer coil across a characteristic length $\xi$ (the blob "size") intermediate between the radius of gyration of the chain and the local Kuhn segment length. Thermic blobs in highly dilute solutions and swollen blobs in semi- dilute solutions have been observed by careful SANS experiments [1] focused on the asymptotic regime of the single chain structure factor $S(q) \propto q^{-1 / \nu}$ where $\nu$ is the scaling exponent. On semi-dilute solutions in good solvent, Farnoux et al. [1] observed a clear change from the excluded volume statistics $(\nu=0.6)$ at length scales characteristic of the interior of the blob to the ideal chain statistics $(\nu=0.5)$ over larger length scales where intramolecular excluded volume forces are screened by the presence of other chains. The size $\xi \approx 1 / q^{*}$ of the "swollen" blob is naturally fixed by the cross-over wave vector $q^{*}$ separating the two regimes.

Dilute solutions of polymers under flow and stretched chains are other notorious examples in which the blob concept has been invoked under the terminology of "shear" blobs and "tensile" blobs respectively. However SANS experiments are more difficult to perform in those cases partly because effects are anisotropic and $S(q)$ thus depends upon the direction of $q$ with respect to the field.

Onuki has conjectured that shear blobs could develop in long linear chains of $N$ bonds in dilute solutions subjected to high shear rates [2]. The shear blob size would correspond to the size of a polymer segment of length $n(n<<N)$ such that the longest relaxation time of this segment is of the order of the inverse shear rate. The mere existence of such blobs has not yet been tested experimentally although the scattering function of polymer solutions under shear has already been probed by SANS experiments in the power law regime [3]. Detecting blobs would require measuring the single chain structure factor of very long and highly stretched chains, a measurement made difficult by a very unfavorable signal to noise ratio at the very low concentrations required to get by extrapolating "infinite dilution" results [4].

In this letter, we report Monte Carlo calculations on the structure factor of very long chains (up to 6000 monomers) stretched at their ends by equal but opposite forces $\mathbf{f}$ which provide direct evidence in favor of the tensile blob concept introduced 20 years ago by Pincus and de Gennes [5, 6]. These authors suggested that when polymers in good solvent are stretched by sufficiently high tensile forces, the coil behaves like a stretched ideal chain formed of tensile blobs as basic units. Inside the blobs, good solvent statistics persists. This picture rests on the assumption that the strong elongation of the chain in the field direction prevents monomers distant along the polymer contour to interact with each other directly. In this picture the blob size $\xi_{T}$ depends on the force intensity as $\xi_{T}=k_{B} T / f$ where $k_{B}$ is the Boltzmann constant and $T$ the temperature.

Macroscopically the onset of tensile blobs above a threshold value of the reduced force $\eta=R_{o} / \xi_{T}\left(R_{O}\right.$ is the unperturbed chain end-to-end distance) results in a change of the elastic behaviour of the chain. For small stretching the elongation $R_{f}$ of the chain, defined as the average projection of the end-to-end vector in the field direction, follows a linear Hooke's law $R_{f}=R_{o} \eta / 3$. In the same $\eta$ regime, fluctuations of the square end-to-end distance parallel and perpendicular to the force remain at the equilibrium value. Above a certain value $\eta^{*}$ one obtains the following power laws

$$
\begin{gathered}
R_{f}=R_{o} B \eta^{2 / 3} \\
\delta R_{\|}^{2}=R_{o}^{2} C_{\|} \eta^{-1 / 3}
\end{gathered}
$$




$$
R_{\perp}^{2}=R_{o}^{2} C_{\perp} \eta^{-1 / 3}
$$

where $B, C_{\|}$and $C_{\perp}$ are model dependent constants (independent of $\mathrm{N}$ and $\eta$ ) and the exponent values $2 / 3$ and $-1 / 3$ arise from the expected linear dependence of $R_{f}, R_{\perp}^{2}$ and $\delta R_{\|}^{2}$ on $N$ at fixed $f$ in this regime. In what follows, we define $R_{\perp}^{2}$ as the fluctuations in any direction perpendicular to stretching.

A direct test of the elastic behavior, as given by eqs. (1, 3) came long ago from the precursory MC simulation of Webman et al. [7] which observed the threshold to be located around $\eta^{*}=2$. More recent MC study [8] has been focused on the cross- over region in the attempt to test renormalization group theory predictions [9].

We concentrate on the high stretching regime and we provide for the first time numerical data for the single chain structure factor. Chain lengths of many thousands beads allow us to provide the first experimental test of Pincus scaling [5]. Data for $q$ perpendicular to the force exhibit a well defined crossover between ideal and self-avoiding chain statistics in a way analogous to the semidilute case studied by Farnoux et al. [1]. In the direction parallel to the field and for large enough q's, the scattering function follows the usual unperturbed chain asymptotic behavior when plotted versus an effective scattering vector taking into account the change in metric due to stretching, as predicted by Pincus [5]. In the low $q$ regime, the generalized Debye function for a continuous gaussian chain [10] very accurately explains all the spectacular features of the scattering function (see figure 4).

Our Monte-Carlo study is based on a polymer model which is a simple necklace of hard spheres connected by rigid bonds taken as length unit. The model is characterized by a hard sphere diameter of $\sigma=0.65$, a value which ensures a fully developed excluded volume statistics [11]. Equilibrium calculations for this model lead to the scaling law: $R_{o}=1.011 N^{0.60}$. We combined a reptation algorithm with the Configurational Bias Monte Carlo (CBMC) method [12] suitably adapted to include the external field influence. A new conformation is attempted by suppressing a segment of $k$ beads from one end of the chain chosen randomly and constructing a new segment of same lenght on the opposite side. Leaving out details about the evaluation of the Rosenbluth weights appearing in the new segment acceptance probability [12], let us just mention here that the set of $p$ trial directions for the single bond reconstruction has been extracted from a probability $\propto \exp \left(f \cos \phi / k_{B} T\right)$ where $\phi$ is the angle between the trial direction and the external force. The number of such trial directions has been chosen in a way to minimize the CPU time between two independent configurations. Two configurations generated during the MC Markov Chain are supposed to be independent when they are separated by $(N / 2 k)^{2}$ successful reptation moves of $k$ beads. Our results are based on statistics of 4000 independent configurations for the chains of length $N<6000$ and $\approx 1200$ independent configurations for the $N=6000$ case. The optimal number of trial directions $p$ is found to increase slightly with the chain lenght (typical value is $p=5$ ). Chain lengths of $N=512,1024,2048$ and 6000 bonds were studied at various stretching forces in the range $2 \leq \eta \leq 40.36$.

Figure 1 shows a Kratky plot of the structure factor of a 6000 bead stretched chain, namely $q^{2} S(q)$ versus $q$ for the scattering vector pointing perpendicularly to the stretching direction. For the three particular values of $\eta$ considered, the crossover from ideal to excluded volume behavior clearly takes place within the $q$ range corresponding to the universal regime of $S(q)$ for unstretched chains. The latter is known to cover a range $3 / R_{g}<q<\lambda^{-1}$ where $R_{g}$ is the equilibrium radius of gyration and $\lambda$ the Kuhn segment length [13]. In the present case such range cover two decades from $q_{\min } \approx 0.04$ up to $q_{\max } \approx 4.0$. In figure 1 , we also report the Debye function in the perpendicular direction [10]

$$
S\left(q_{\perp}\right)=\frac{2(N+1)}{X_{\perp}^{2}}\left(e^{-X_{\perp}}-1+X_{\perp}\right)
$$

where $X_{\perp}=q_{\perp}^{2} R_{\perp}^{2} / 2$ and values of $R_{\perp}^{2}$ are directly obtained in the simulation. The Debye function is seen to follow very closely the chain structure factor up to the crossover region beyond which all three experimental curves converge towards a unique power law behavior in $q^{1 / 3}$ (represented in the figure as a straight line) which is reminiscent of the equilibrium universality $S(q) \propto q^{-5 / 3}$. The observed asymptotic cross-over wave vectors $q^{+}(\eta)$ (taken as the intersection value between the excluded volume power law and the Debye functions) should be related to the blob size $\xi_{T}$. Indeed one can easily verify that $q^{+}(\eta)=4.5 / \xi_{T}(\eta) \approx 2 \pi / \xi_{T}(\eta)$.

An important issue is about the universality of $S(\mathbf{q}, \mathbf{f}, N)$. In figure 2, we have plotted the form factors $S(q) /(N+1)$ for various chain lengths at the same reduced force $\eta=7$ versus $q R_{0}$. In each specific direction we get a distinct universal function, which suggests an extension of the well known equilibrium universality of $S(q)$ towards $S\left(q_{\perp}\right)=(N+1) f_{\perp}\left(q_{\perp} R_{0}, \eta\right)$ and $S\left(q_{\|}\right)=(N+1) f_{\|}\left(q_{\|} R_{0}, \eta\right)$. Instead if we compare $S(q)$ data for different chain lengths at the same absolute force intensity $f$, all curves are found to merge for $q>q^{*}$ as it should be.

Along the lines of previous calculations, [7, \&] we have analyzed the end-to-end vector statistics of stretched chains and we confirm that, in the high stretching regime, the elongation as well as the squared fluctuations follow the universal laws eqs.(11, 2, (3) in terms of $\eta$. The model dependent parameters are $B=0.46, C_{\|}=0.31$ and $C_{\perp}=0.46$. 
Figure 3 shows the systematic evolution of $S\left(q_{\perp}\right)$ and $S\left(q_{\|}\right)$as $\eta$ increases for $N=6000$. In the direction of the force we observe the appearance of a shoulder which becomes more and more pronounced as the chain extends. As $\eta$ gets even larger, oscillations develop beyond the initial decay. The origin of this behavior can easily be understood by comparison with the structure factor for a rigid rod of length $L$ with $N+1$ scatterers uniformly distributed which is, for $q$ parallel to the rod [2],

$$
S(q)=\frac{2(N+1)}{(q L)^{2}}(1-\cos (q L))
$$

In figure 3, the comparison with the rigid rod is made for $\eta=40.36$, using $L=R_{o} B \eta^{2 / 3}$ (see eq.(1)). At small $q$ the chain can be described as a rod of fluctuating length, a point not totally surprising if we note that for $N=6000$ and $\eta=40.36$, the average extension is one sixth of the polymer contour length! The rigid rod model becomes obviously more and more inaccurate for increasing $q$, that is on the length scales where fluctuations start dominating the systematic effects. Further, we note that beyond $q=10 / \xi_{T}$, indicated by vertical bars in the figure, the structure factor remains isotropic.

Figure 4 shows the structure factor for $q_{\|}$in the $N=6000$ case for four values of $\eta=0.00,7.00,13.34,40.36$. Data for $\eta \neq 0$ are also reported versus the effective wave vector $\tilde{q}$ defined as

$$
\tilde{q}^{2}=q^{2}+\frac{4}{\xi_{T}^{2}} \cos ^{2} \theta
$$

where $\theta$ is the angle between the observation direction and the external field [5]. For $\cos \theta \neq 0, \tilde{q}$ is a non linear combination of the bare $q$ vector and a measure of the systematic stretching effect. For $q \gg \xi_{T}^{-1}, \tilde{q} \simeq q$ and we recover the typical power law of the equilibrium scattering function. In an intermediate region $q^{* *} \leq q \leq 10 / \xi_{T}$ the curve bending of $S\left(q_{\|}\right)$produced by the external force and directly related to the presence of a finite systematic stretching, is compensated by plotting $S\left(q_{\|}\right)$versus $\tilde{q}$. This brings back the data on the equilibrium power law [5]

$$
S\left(q_{\|}\right) \sim \tilde{q}^{-1 / \nu}
$$

Finally in the region $q \leq q^{* *}, \tilde{q}$ becomes almost constant so that the data exhibit an apparent divergence when plotted against $\tilde{q}$.

As for the perpendicular direction, the continuous gaussian chain model can be exploited to explain the $S\left(q_{\|}\right)$at low $q$. The generalized Debye function is 10

$$
S\left(q_{\|}\right)=2(N+1) \Re\left(\frac{e^{-X_{\|}}-1+X_{\|}}{X_{\|}^{2}}\right)
$$

where $X_{\|}$is a complex variable defined as

$$
X_{\|}=q_{\|}^{2} \frac{R_{o}^{2}}{2} C_{\|} \eta^{-1 / 3}+i q_{\|} R_{o} B \eta^{2 / 3}
$$

In figure 4 we report the Debye behavior (dashed lines) for the three values of $\eta$ and for values of the elongation and the parallel fluctuation given by the observed power laws behaviors of eqs.(11.22). The agreement between the ideal chain prediction and the experimental data is amazingly good up to $q$ values close to $q^{* *}$.

In conclusion we have simulated long linear chains stretched by a uniform force. We have studied the strong stretching regime where tensile blobs dominate the elastic behavior of the chains. We have performed a careful analysis of the chain scattering function and thanks to the large dimension of our chains we have been able to directly observe the blob signature as a cross- over in the universal behavior of the scattering function in the direction orthogonal to the force. We have provided the first "experimental" evidence that Pincus scaling in terms of an effective wave vector [5] describes accurately the entire excluded volume region of the scattering function (inside the blobs). At larger length scales the generalized Debye function beautifully explains the scattering data, in particular at very high extension when the chain behaves as a collection of blobs aligned as a linear rod. The picture of an ideal chain of $N_{b}$ blobs of size $\xi_{T}$ stretched by a force $f$ is however only qualitatively supported by our data. Such a model requires $R_{\perp}^{2}=\delta R_{\|}^{2}=(1 / 3) N_{b} \xi_{T}^{2}$ together with $R_{f}=(1 / 3) N_{b} \xi_{T}^{2}\left(f / k_{B} T\right)$ which according to the observed behaviour of eqs. (1), 2, 3) demands $C_{\perp}=C_{\|}=B$. In our results, $C_{\perp}=B$ is indeed observed but $C_{\|} \approx 2 / 3 C_{\perp}$.

The relevance of our results with respect to the experimental situation is threefold: first, it should be noted that single chain stress-strain curves are nowadays directly measured in beautiful experiments on single DNA molecules [14]. 
However, similar experiments on flexible uncharged single polymers in good solvent still appear unfeasible. The second and more traditional experimental source of information on stretched chains would be scattering measurements on highly diluted solvated gels. However, well known network heterogeneities of such samples will transmit stress on junction points in a uncontroled way, making it very difficult to relate observed spectra to single chain statistics. Finally, it appears as most likely that the first experimental test of the features of the chain structure factor discussed in the present letter will be provided by SANS experiments on sheared polymer solutions.

We have the pleasure to thank G. Destrée for unvaluable technical assistance. We thank P. Lindner, D. Richter and J. Titantah for illuminating discussions. We benefited from a generous INFM computer grant on the CRAY-T3D parallel computer at Cineca, Bologna (Italy).

[1] B. Farnoux et al., J. Physique 39, 77 (1978).

[2] A. Onuki, J. of Phys. Soc. Japan 54, 3656 (1985).

[3] P. Lindner, in Neutron, X-ray and Light Scattering, edited by P. Lindner and T. Zemb (Elsevier science Publishers, 1991).

[4] P. Lindner, L. Mayer, C. Pierleoni, and J.-P. Ryckaert, ILL Report Exp 9-11-383 (1997).

[5] P. Pincus, Macromolecules 9, 386 (1976).

[6] P.-G. de Gennes, Scaling Concepts in Polymer Physics (Cornell University Press, Ithaca,N.Y., 1979).

[7] I. Webman, J. Lebowitz, and M. Kalos, Phys Rev. A 23, 316 (1981).

[8] M. Wittkop, J.-U. Sommer, S. Kretmeier, and D. Gritz, Phys. Rev. E 49, 5472 (1994).

[9] Y. Oono, T. Ohta, and K. Freed, Macromolecules 14, 880 (1981).

[10] H. Benoit et al., Macromolecules 8, 451 (1975).

[11] A. Ladd and D. Frenkel, Macromolecules 25, 3435 (1992).

[12] D. Frenkel and B. Smit, Understanding Molecular Simulation (Academic Press, San Diego, 1996).

[13] M. Rawiso, R. Duplessix, and C. Picot, Macromolecules 20, 630 (1987).

[14] S. Smith, L. Finzi, and C. Bustamante, Science 258, 1122 (1992).

FIG. 1. $\mathrm{N}=6000$. Kratky plot of $S(q) q^{2}$ versus $q$ for $\eta=7$ (circles), 13.34 (squares), 40.36 (crosses). Dashed curves are the predictions of the Debye function and the straight line represent the excluded volume behavior $S(q) q^{2} \sim q^{1 / 3}$.

FIG. 2. Universal behavior of $S(q) /(N+1)$ versus $q R_{o}$ at $\eta=7$. Various chain lengths are shown for both $q$ parallel (lower curve) and perpendicular (upper curve) to the external field. $\mathrm{N}=512$ (circles), 1024 (squares), 2048 (crosses) and 6000 (stars). Equilibrium behavior for $\mathrm{N}=1024$ is given by the thick line.

FIG. 3. $\mathrm{N}=6000 . S(q)$ for $q$ perpendicular (upper curves) and parallel (lower curves) to the field for $\eta=7$ (circles), 13.34 (squares), 40.36 (crosses) versus the bare wave vector $q$. The continuous line is the results of the rigid rod model mentioned in the text.

FIG. 4. $\mathrm{N}=6000 . S\left(q_{\|}\right)$for $\eta=7$ (circles), 13.34 (squares), 40.36 (crosses) versus the bare wave vector $q$ (lower curves) and the rescaled wave vector $\tilde{q}$ (upper curves). For each value of $\eta$, the lower boundary $q^{* *}(\eta)$ for the validity of Pincus' scaling is indicated by a vertical bar. Dashed curves are the predictions of the Debye function. Note that they are very accurate up to $q^{* *}(\eta)$ which is therefore the crossover from ideal to excluded volume behavior. 


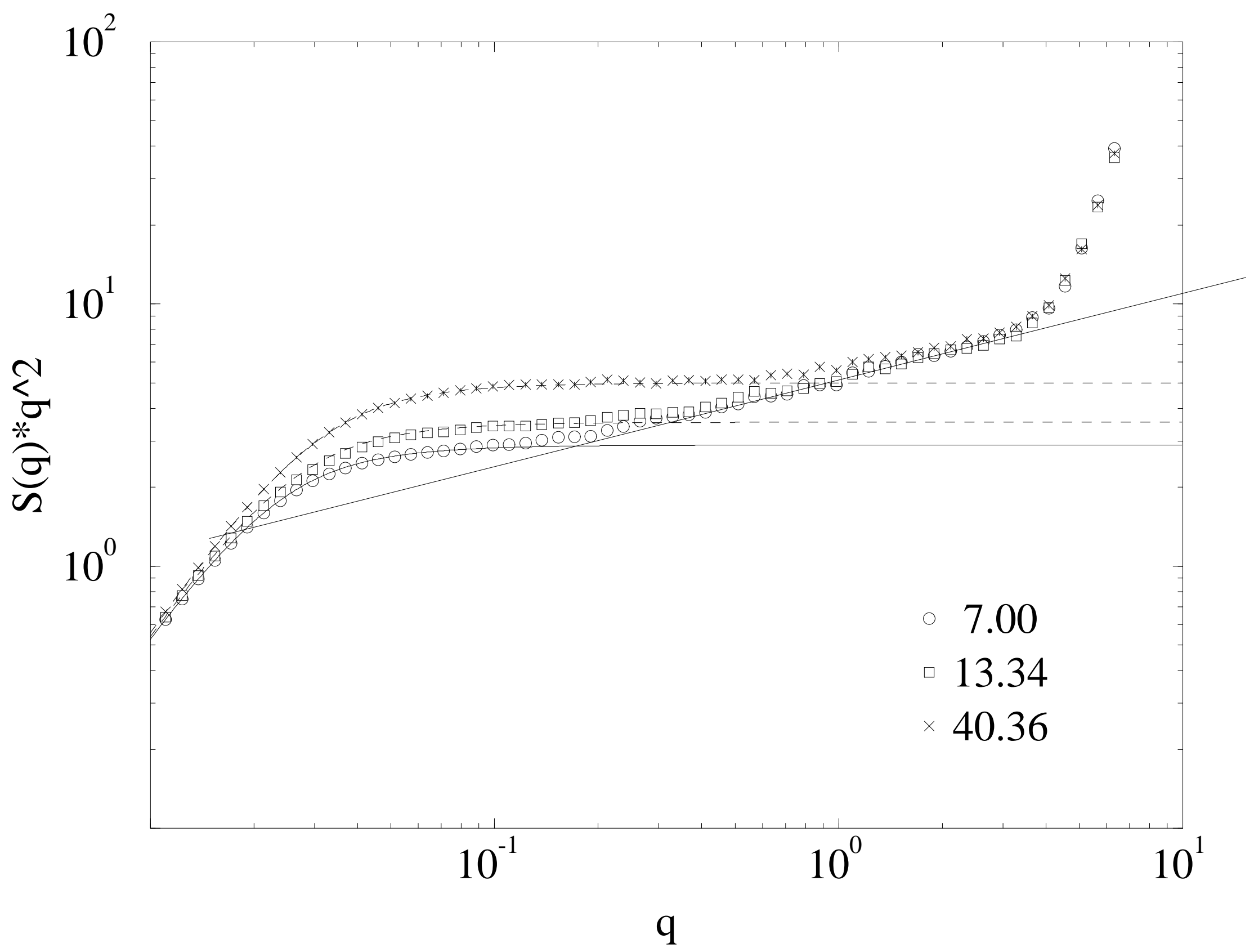




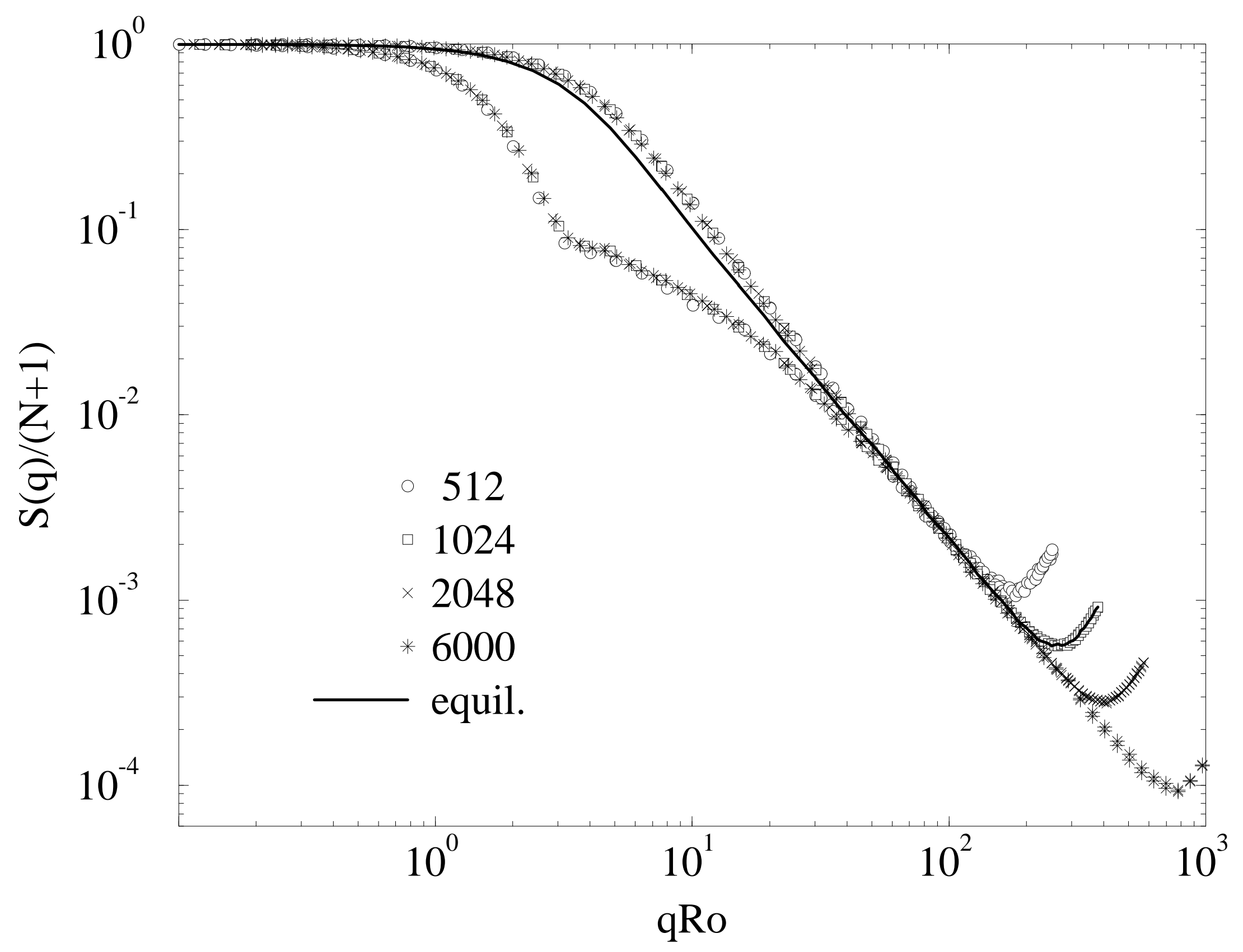




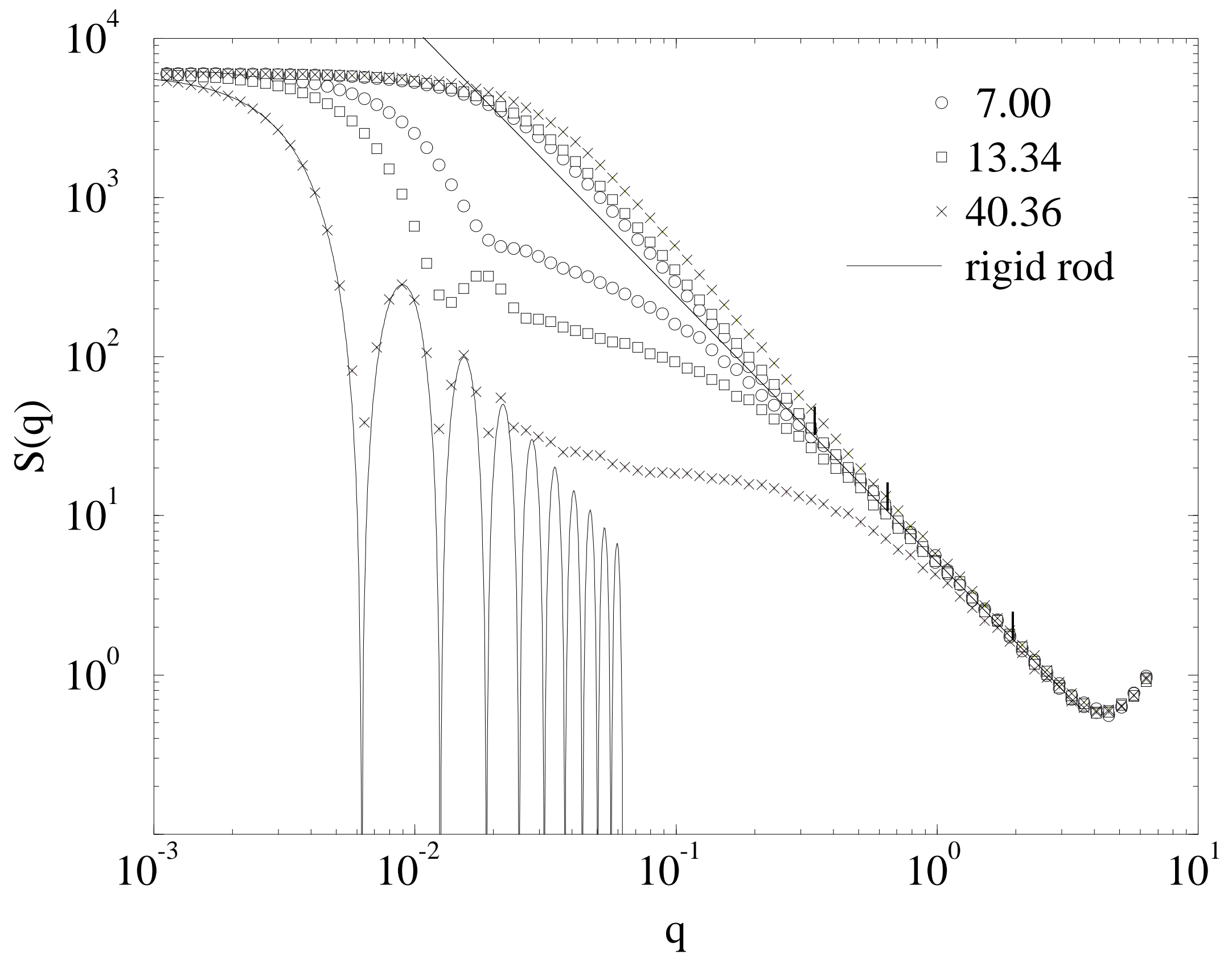




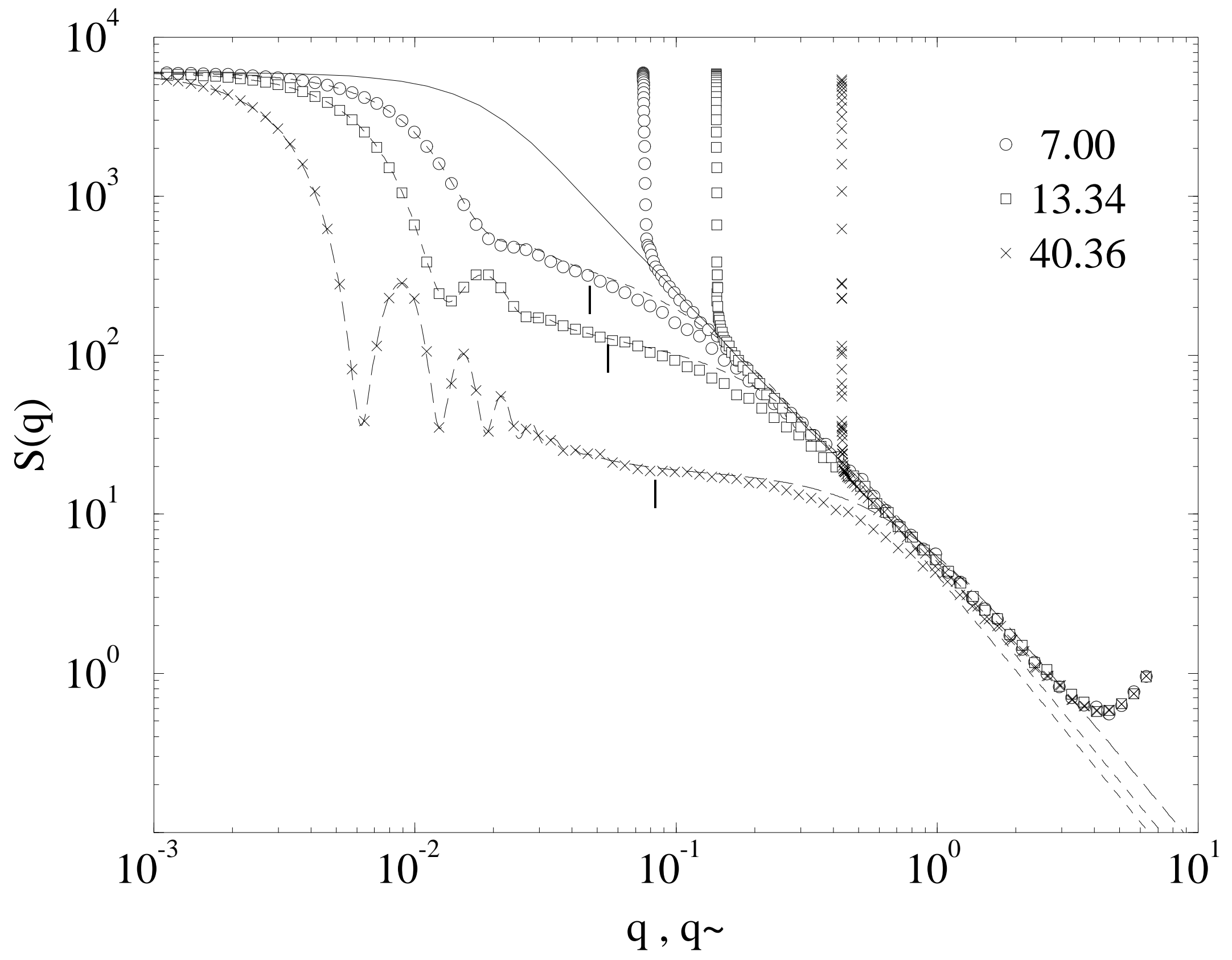

